ESJ Social Sciences

\title{
Las TIC y el e-learning y su influencia en el proceso de enseñanza aprendizaje del idioma Inglés en estudiantes de educación superior
}

\section{Silvania Gabriela Herrera Martinez}

Licenciada en ciencias de la educación mención Lengua Inglesa y Lingüística

Directora Del Departamentos De Idiomas Del ISTSB

Instituto Superior Tecnológico Simon Bolívar

William Wilfrido Suáres Ureña

Ingeniero en sistemas computacionales

Magister en sistemas de información gerencial

Instituto Superior Tecnológico Enrique Noboa Arízaga

María Fernanda Núñez López

Ingeniero en contabilidad y auditoría

Contadora independiente

Deysi Lucía Damián Tixi

Licenciada en Ciencias de la Educación Profesora de Inglés

Magister en Lingüística y Didáctica en la Enseñanza de Idiomas Extranjeras

Escuela Superior Técnica de Chimborazo en el Centro de Idiomas

\section{Jazmin Estela Benites Mero}

Licenciada en Ciencias de la Educacion Mencion Lengua y Lingüística

Coordinadora del Centro de Idiomas

Instituto Superior Tecnologico Juan Bautista Aguirre

Doi:10.19044/esj.2021.v17n23p96

Submitted: 20 June 2021

Accepted: 22 July 2021

Published: 31 July 2021
Copyright 2021 Author(s)

Under Creative Commons BY-NC-ND

4.0 OPEN ACCESS

Cite As:

Herrera Martinez S.G., Suáres Ureña W.W., Núñez López M.F., Damián Tixi D.L. \& Benites Mero J.E. (2021). Las TIC y el e-learning y su influencia en el proceso de enseñanza aprendizaje del idioma Inglés en estudiantes de educación superior. European Scientific Journal, ESJ, 17(23), 96.https://doi.org/10.19044/esj.2021.v17n23p96

\section{Resumen}

En este artículo se reflexiona desde el punto de vista conceptual, sobre la influencia que las Tecnologías de la Información y la Comunicación (TIC) y el impacto que éstas han generado en los procesos de enseñanza/aprendizaje. La educación superior en Colombia ha venido transformándose en las 
recientes décadas, con miras a responder de manera decidida a los requerimientos de una sociedad renovada, que busca contar con profesionales más hábiles, que gestionen eficientemente procesos administrativos y académicos en Instituciones de Educación Superior en estos tiempos de globalización económica. La metodología utilizada en este estudio se enmarca en la revisión documental de investigaciones y publicaciones efectuadas en los últimos diez años. Los resultados apuntan a que las TIC han aportado considerablemente a diferentes aspectos neurálgicos, en el contexto educativo y que las aplicaciones en los procesos pedagógicos son cada vez mayores.

Palabras claves: TIC, E-Learning, Enseñanza, Inglés, Educación Superior

\section{Las TIC y el e-learning y su influencia en el proceso de enseñanza aprendizaje del idioma Inglés en estudiantes de educación superior}

\section{Silvania Gabriela Herrera Martinez.}

Licenciada en ciencias de la educación mención Lengua Inglesa y Lingüística

Directora Del Departamentos De Idiomas Del ISTSB

Instituto Superior Tecnológico Simon Bolívar

William Wilfrido Suáres Ureña

Ingeniero en sistemas computacionales

Magister en sistemas de información gerencial

Instituto Superior Tecnológico Enrique Noboa Arízaga

María Fernanda Núñez López

Ingeniero en contabilidad y auditoría

Contadora independiente

Deysi Lucía Damián Tixi

Licenciada en Ciencias de la Educación Profesora de Inglés

Magister en Lingüística y Didáctica en la Enseñanza de Idiomas Extranjeras

Escuela Superior Técnica de Chimborazo en el Centro de Idiomas

Jazmin Estela Benites Mero

Licenciada en Ciencias de la Educacion Mencion Lengua y Lingüística

Coordinadora del Centro de Idiomas

Instituto Superior Tecnologico Juan Bautista Aguirre

\section{Abstract}

This article reflects from the conceptual point of view, on the influence that Information and Communication Technologies (ICT) and the impact they have generated in the teaching / learning processes. Higher education in 
Colombia has been transforming in recent decades, with a view to responding decisively to the requirements of a renewed society, which seeks to have more skilled professionals, who efficiently manage administrative and academic processes in Higher Education Institutions in these times of economic globalization. The methodology used in this study is part of the documentary review of research and publications carried out in the last ten years. The results suggest that ICTs have contributed considerably to different neuralgic aspects in the educational context and that the applications in pedagogical processes are increasing.

Keywords: ICT, E-Learning, Teaching, English, Higher Education

\section{Introduction}

La sociedad actual, retratada por un escenario de globalización y por el rápido desarrollo de las Tecnologías de la Información y la Comunicaciones (TIC), ha incentivado a la creación de mecanismos y/o componentes para la integración de diversos avances tecnológicos en diferentes ámbitos monetarios y sociales (Baller, Dutta, \& Lanvín, 2016). El ámbito escolar no se aleja de esta realidad, incluyendo las TIC como una maravilla ineludible con un alto índice en el ámbito instructivo, por la necesidad de avanzar en el desarrollo y la innovación, en el que se deben delinear las Instituciones de Educación Superior (IES), para la operacionalización de los considerables capacidades (Sanz \& Crissien-Borrero, 2018).

De esta manera, el marco de enseñanza-aprendizaje se ajustará en general a estos cambios, a través de la elaboración de estrategias instructivas que incluyan estas herramientas en los entornos de aprendizaje (Herrera, 2015), Desde un punto de vista técnico, las TIC se fundamentan en: los referentes relativos a las tecnologías de la comunicación (medios de radiodifusión, televisión y telefonía, entre otros) y las tecnologías de la información, que engloban los mecanismos en los cuales se almacenan y gestionan datos e información (Ausín, Abella, Delgado, \& Hortigüela, 2016).

Desde una perspectiva mundial, las TIC se relacionan con los componentes y herramientas a través de los cuales se pueden preparar, almacenar y distribuir datos de diferentes fuentes. Convertirse en un componente particular de innovación que retrata la cultura actual y que está afectando el aprendizaje de las personas y las formas habituales de difusión de información (Zempoalteca, Barragán, \& González, 2017). En particular, en el entorno instructivo, las TIC se relacionan con un conjunto de aparatos de ayuda mecánica para que los educadores fortalezcan la medida de aprendizaje de la instrucción en todos los grados de formación (Arancibia, Contreras, \& Soto, 2010). 
Según lo indicado por la Organización de las Naciones Unidas para la Educación, la Ciencia y la Cultura (UNESCO), la mecha de las TIC en la educación avanzada se utiliza para mejorar la exhibición de materiales, compartir contenido y mejorar la correspondencia entre los animadores en el ciclo instructivo, avanzar y fomentar la investigación y trabajar con ciclos regulatorios institucionales, entre otros (UNESCO, 2013). En muchas IES, las TIC se han adoptado como un método para el cambio y la mejora de las medidas de instrucción de aprendizaje en el marco escolar, luego de sacar a la luz los problemas de las limitaciones y elementos utilizables (Koh, 2015). Sea como fuere, la utilización y ejecución de las TIC no es inseparable del logro asegurado en cuanto a la creación de sistemas productivos para la creación y difusión de información. De ahí que sea fundamental avanzar en espacios con las condiciones adecuadas para el mayor aprovechamiento de estos aparatos (Ausín, Abella, Delgado, \& Hortigüela, 2016).

\section{Las TIC en el mundo}

La sociedad a nivel mundial se ha visto bastante afectada por el impacto que generan las TIC en la totalidad de las mediciones; Los cambios sociales, monetarios, instructivos y sociales han hecho concebibles nuevas situaciones donde el individuo ha fortalecido cada uno de los puntos de vista que se han propuesto buscando un despliegue más agradable y estable como lo indican los supuestos de vida (Jin y Cho, 2015). Asimismo, las perspectivas de utilidad y administración han cambiado significativamente, llegando a niveles de grandeza nunca vistos, donde usuarios y clientes obtienen colosales ventajas derivadas de los avances logrados tanto en tecnología como en datos (Pérez \& Olguín, 2018).

Según investigaciones de diferentes enfoques realizadas por diferentes organizaciones, los impactos más destacados de las TIC a nivel mundial tienen que ver con la universalización de las cosas, ya que en la medida en que las tecnologías y la dispersión de datos se han desarrollado a raíz de avances conocidos, ha sido factible interconectar el mundo entero en todos los espacios, fomentando focos de encuentro progresivamente líquidos, dinámicos y serios, lo que sin duda ha cultivado la prosperidad y el desarrollo financiero de algunas redes (Asongu, 2017).

En temas de sectores particulares existen numerosos modelos a los que se puede hacer referencia, ya que desde los masivos sistemas de transporte, bienestar, creación de productos e incluso proyectos escolares, en igualdad de condiciones, se han visto mejoras y avances que han hecho concebible abreviar distancias, recortar tiempos de consideración, realizar procedimientos no contemplados y avanzar en la cercanía entre comunidades urbanas y partidos creados, lo que sin duda ha hecho concebible el lanzamiento de una 
sociedad virtual donde la presencia real no es fundamental para hacer giros de eventos o ciclos para acabar (Baldassar, 2016).

\section{Las TIC en el contexto educativo}

En el ámbito instructivo, las TIC se han incorporado últimamente, con especial ímpetu específicamente en los procesos de enseñanza/aprendizaje. Esto ha desencadenado una progresión de cambios en la cultura actual, identificada con la producción de condiciones de aprendizaje menos inflexibles, en las que el factor fugaz o área espacial está a todos los efectos excluido. Las TIC han ocupado espacios que antes no se pensaban en la formación convencional (Cabero J., 2010).

En esta situación, es pertinente hacer referencia a la importancia de los locales avanzados, un término escrito por Prensky (2001), quien sostiene que el diseño de razonamiento no es del todo el mismo que el de los individuos que no experimentaron la infancia en el clima informatizado actual. En consecuencia, esta era de lugareños avanzados probablemente piensa de manera única en contraste con el resto de las edades pasadas. En este sentido, para los locales informatizados, la implicación con la utilización de las TIC plantea nuevos requerimientos en las medidas de aprendizaje, que el marco instructivo pretende atender. El suplente contemporáneo utiliza la innovación como instrumento esencial en el escenario individual y espera que también lo sea en el instructivo. En cualquier caso, no debería ser normal que todos los locales informatizados estén equipados para recibir información interna y externa sobre el uso de las TIC de forma natural, ya que las habilidades e información sobre innovación cambian según lo indican los atributos específicos de cada persona (Bello, 2018).

Tomando como premisa la forma en que los locales computarizados tienen habilidades más destacadas en la utilización de activos mecánicos, y que la perspectiva se ajusta parcialmente a ellos, el educador se convierte en un guía cuyo objetivo fundamental es trabajar con la conveniencia de los métodos innovadores, para propósitos instructivos útiles. Esta utilización puede percibirse, en la educación avanzada, como el intercambio competente de información que insta al estudiante a comprender y crear nueva información (Asongu, 2017).

\section{Aplicaciones de las TIC en el ámbito educativo}

Contemplando las hipótesis referidas hasta ahora, se ha demostrado que las técnicas de instrucción / aprendizaje han avanzado a espacios de mayor adaptabilidad, desarrollo e innovación. Asignar al educador la tarea de planificar mejores enfoques para avanzar en el nombramiento de información por parte de los estudiantes suplentes y para tratar diferentes métodos de correspondencia con los estudiantes suplentes (Albion, 2015). 
Como se puede encontrar en la figura 1, la conciliación en un plano similar de puntos de vista, por ejemplo, la adaptabilidad, el avance y la imaginación, hacen avanzar una situación de uso de metodologías académicas mucho más extensas que las utilizadas hasta años y años antes; potenciando ciclos relacionados y dando espacios, ya no pensados, que trabajarán con la comunicación entre los diferentes animadores. Es imperativo resaltar que para el fortalecimiento de las TIC en escenarios instructivos, como aparatos que avanzan en el desarrollo de las medidas muestrales, las metodologías no deben ser aplicadas exclusivamente desde la metodología académica, también deben generar situaciones de actividad en las que se potencie la asignación de avances. En el modelo instructivo, en la creación de materiales computarizados, la preparación de educadores para la utilización significativa de las TIC y la exploración identificada con TIC e Innovación (Marín, Inciarte, \& Hernández, 2017).

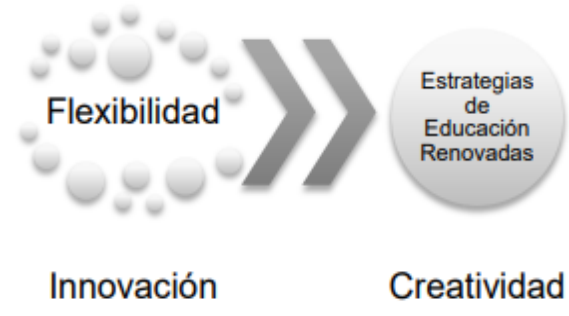

Figura 1. Estrategias Enseñanza /aprendizaje utilizando TIC Fuente: (Albion, 2015)

Las TIC ofrecen los instrumentos para la producción de tales situaciones y potencian la planificación y ejecución de nuevas técnicas académicas. Un ejemplo de esto es la utilización de Ambientes Virtuales de Enseñanza - Aprendizaje (EVEA), operacionalizados a través de etapas virtuales, que permiten la comunicación entre instructores y estudiantes suplentes en torno a una sustancia en particular, y utilizando estrategias y métodos claros, para mejorar las habilidades y producir información en un formato de manera significativa (López \& Hernández, 2016). En un orden específico de pensamientos, la variedad de dispositivos que existen hoy, por ejemplo, teléfonos celulares y tabletas, pueden coordinarse sin mucho esfuerzo en este entorno avanzado, para reproducir espacios creativos que animan el aprendizaje.

\section{E-Learning en la educación superior.}

Los autores Zempoalteca, et al., (2017) caracterizan el E-Learning como una pieza de educación a distancia que ejecuta avances utilizando estrategias simultáneas y poco convencionales para introducir datos, utilizando más de un método para la correspondencia, ya sea video, sonido o 
movimientos para ver, hacer o mejorar los materiales del espectáculo. El plan incorpora ángulos instructivos, innovadores y autorizados.

En esta línea, el creador Álvarez (2012) hace referencia a que los LMS tienen una progresión de cualidades Número de clientes, tipo de trabajadores, bases de información, determinación de E-Learning (AICC y SCORM), creación de contenidos y el tablero, orientación en Salas reales y virtuales, trabajo conjunto (charlas, tertulias, mail, tertulias de trabajo), multilenguaje, que permiten ayudar a los instructores y suplentes en los ejercicios escolares.

Además, Alsabawy, Cater-Steel (2013) presentan que la evaluación de los marcos de E-Learning es fundamental para medir la prosperidad, así como para la propia administración y mejora. Reflexionan sobre un modelo de evaluación que incorpora: administraciones marco, utilidad, cumplimiento, valor del cliente y estima de la asociación. Ausín, et al,. (2016) Presentan un modelo de evaluación dependiente de la Web no concurrente y las sugerencias, considerando el cumplimiento y ejecución global de los estudiantes suplentes e instructores, la asociación y la junta de aprendizaje, cooperación y comunicación entre ellos.

Simultáneamente, se introducen algunos escenarios para la evaluación del E-Learning: ángulos especializados y útiles, efecto de los ciclos psicológicos, influencia en los ciclos de cooperación y reconocimiento del ELearning. El creador Deveci (2016) habla de que para evaluar y conocer la realización del E-Learning, se debe ponderar los materiales y la utilización de aparatos especializados, la comunicación entre estudiantes suplentes, el plan del clima educativo, la conducta hacia el curso y el sustancia del curso en la interacción educativa.

La felicidad con respecto al curso se puede romper adicionalmente con el reconocimiento de los materiales, la apreciación por el contenido y la educación, los aparatos de contenido dependientes de las necesidades de los estudiantes, la cooperación simultánea y poco convencional, la disposición de los estudiantes hacia el aprendizaje electrónico, la variedad en el evaluación y práctica de actividades, clima del plan, inspiración y moderación del aprendizaje. Alsabawy, Cater-Steel (2013) planificaron y evaluaron un modelo para la administración de la medida de E-Learning, asumiendo que existe una conexión entre la organización y control de ciclo con resultados de aprendizaje.

Marshall (2012) especifica que el modelo de desarrollo de E-Learning (EMM) ofrece un sistema de mejora de la calidad que estima a las organizaciones y las impulsa a lograr un E-Learning manejable. Las medidas de EMM se evalúan completamente de acuerdo con la transmisión, la disposición, la definición, el tablero y el avance. El instrumento utiliza registros institucionales y pruebas de cursos, al igual que el límite del establecimiento en la ayuda operativa del E-Learning. 
Los objetivos del EMM son relevar y diseccionar la evaluación y conectar límites explícitos y límites generales, así como insuficiencias para reconocer los procedimientos para que los jefes de las fundaciones realicen cambios, ayudando a los establecimientos a desarrollarse. Las tecnologías de la información y correspondencia (TIC) se han convertido en instrumentos cruciales en diferentes partes de la vida actual. Es difícil pensar en el mundo sin PC que permitan el acceso a Internet, organizaciones informales y todos los métodos virtuales de correspondencia que funcionan con contacto y esfuerzo coordinado entre individuos (Marshall, 2012).

En esta línea, el surgimiento de nuevos avances y métodos de trabajo ha hecho que los expertos, y en particular los instructores entre ellos, fomenten habilidades y capacidades para utilizar adecuadamente los dispositivos mecánicos. Teniendo en cuenta que la educación superior se enfrenta a la prueba de preparar expertos y residentes aptos para afrontar las demandas del lugar de trabajo de la sociedad del siglo XXI, Albion (2015), insiste en que una de las dificultades de los colegios es preparar individuos equipados, para lo cual los instructores actuales deben tener una progresión de cualidades que les haga desempeñarse aceptablemente en clase y en la vida diaria (Baldassar, 2016).

La realidad actual tiene una prueba increíble debido a la pandemia de COVID-19, la Organización Mundial de la Salud (OMS), ha anunciado la infección como una pandemia por la velocidad de propagación y el gran tamaño de enfermedad, posteriormente, en muchas naciones del En el mundo, los gobiernos han venido tomando medidas para prevenir la propagación, siendo en el marco instructivo la conclusión de fundamentos instructivos. Según el informe presentado por la UNESCO en abril de 2020, el rápido efecto de COVID-19 ha influido en alrededor del 70\% de la población de estudiantes suplentes del mundo (Pérez \& Olguín, 2018).

Como opción frente a esta realidad, los medios informatizados se han convertido en un instrumento imprescindible para la escolarización, con el único objetivo de seguir aprendiendo medidas prácticas. En la mayoría de las naciones (29 de las 33), se han establecido tipos de progresión de estudios en diferentes modalidades a distancia. Entre ellos, 26 naciones llevaron a cabo tipos de aprendizaje basado en la web y 24 establecieron técnicas de aprendizaje a distancia en modalidades desconectadas, recordando 22 naciones para las cuales se ofrece educación a distancia en las dos modalidades (desconectado y en la web), 4 que consultan solo en línea y 2 con modalidades simplemente desconectadas (CEPAL-UNESCO, 2020).

Siguiendo con el informe elaborado por la Comisión para América Latina y el Caribe, entre las modalidades de aprendizaje a distancia en línea utilizadas en todo el mundo, destaca la utilización de etapas virtuales de aprendizaje no concurrente, utilizadas en 18 países, mientras que solo 4 países 
ofrecen clases en vivo (Bahamas, Costa Rica, Ecuador y Panamá). Así, entre los tipos de educación a distancia desconectada, 23 naciones transmiten proyectos instructivos a través de medios convencionales como la radio o la televisión (CEPAL-UNESCO, 2020).

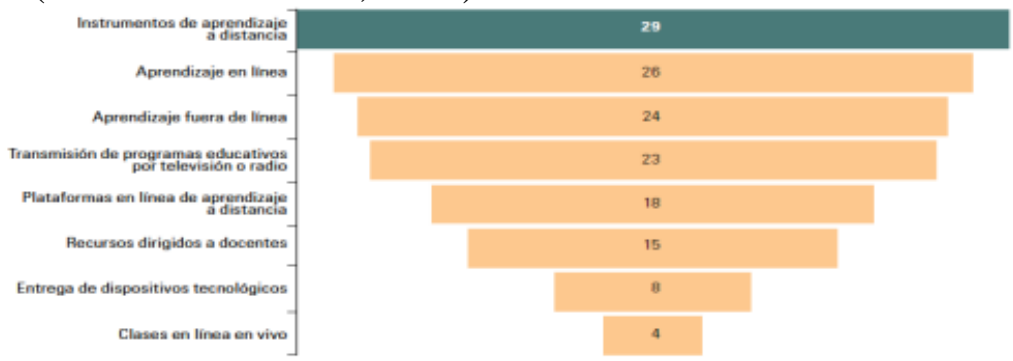

Ilustración 1: Estrategias de continuidad de estudios en modalidades a distancia.

Fuente: (CEPAL-UNESCO, 2020)

Es imprescindible precisar que según Horacio, et al., (2020) los arreglos recibidos por las naciones se han centrado principalmente en la trasmisión de sustancia en correspondencia con el plan educativo escolar. La oportunidad se ha basado en los límites de la base anterior y la sustancia accesible para fabricar un modelo de instrucción a distancia, rápido y en crisis (Hodges, 2020).

Esta metodología de aprendizaje a distancia se está completando en un tiempo y velocidad fenomenales, y se describe por tener una combinación de arreglos y medios originales (materiales impresos, radio y televisión y una segunda era (etapas, los marcos ejecutivos de descubrir cómo) para transmitir contenido y mantener cierto grado de conexión entre las escuelas y los estudiantes suplentes. Estas diversas modalidades requieren un respaldo constante, una orientación, que ha sido muy heterogénea entre los marcos (Horacio, Ortiz, \& Bergamaschi, 2020).

Una de las modalidades de instrucción que utiliza datos e innovaciones de correspondencia es la escolarización combinada, en la que la combinación entre el modelo vis-à-vis y el modelo e-Learning (este tipo de educación basada en la web permite al cliente conectarse con el material utilizando diferentes Dispositivos PC). Uno que complementa y orquesta dos opciones que, hasta un par de años antes, parecían estar en conflicto para muchos: prepararse cara a cara con prepararse a través de las TIC. En este nuevo tipo de preparación, dispositivos especializados como el correo electrónico, los registros de apropiación o el Chat plantean la necesidad de preparar a los dos instructores y suplentes para una utilización suficiente, razonable y significativa de los mismos (Cabero, Llorente, \& Román, 2015).

En vista de lo anterior, en estos días no se trata, en este punto se habla de si coordinar los avances de datos y correspondencia - TIC - en las 
Instituciones de Educación Superior (IES), sino cómo hacerlo de manera que se potencie lo que debería ser posible. manejarse sin ellos, considerando avanzar, complementar y realizar grandes actos adaptables de instrucción ojo a ojo, un número significativo de los cuales generalmente son respaldados por diferentes medios que no son PC, al incluir ejercicios de aprendizaje que utilizan las TIC, independientemente de si dichos ejercicios son o no ojo, semi - cara a cara o de lejos. En cualquiera de estas situaciones, el educador sigue siendo un componente esencial, como coordinador y guía de las medidas de aprendizaje progresivamente autónomas y auto controladas y como orquestador de los activos instructivos que la fundación e internet ponen al alcance de los estudiantes (Cabero, 2010).

Esta metodología de E-Learning que respalda la medida de educación y aprendizaje explícitamente a las de carácter universitario, tiene como quizás el mejor impedimento la ausencia de ingreso a PC en regiones del país, huecos avanzados, carencias subyacentes, competencia computarizada en instructores y delegados. A esto se suma el gasto humano que esta diferencia de situación desde el aula hasta el PC implica para algunos docentes que deben acomodar los factores apremiantes del mundo experto con el mundo familiar. Todo lo anterior nos permite considerar como motivación de esta exposición, examinar la utilización y desarrollo del E-Learning en la educación avanzada, para lo cual se realizó un ciclo de relevamiento de fuentes narrativas innatas a la asignatura, artículos explícitamente lógicos, trabajos de evaluación, con lo que se ampliará la información sobre este espacio de conocimiento (CEPALUNESCO, 2020).

Aprender inglés se ha convertido en una necesidad para el marco instructivo chileno. Las entradas sociales y sociales que se abren para las personas que se comunican en este idioma se suman a las financieras, ya que el orden del inglés les permite obtener más vacantes de investigación y trabajo. Frente a esta exhibición, y con la plena intención de lograr la satisfacción del individuo de manera básica, la utilización del inglés ha sido considerada como una de las necesidades que orientan los acercamientos del público chileno. El Ministerio de Educación ha realizado el ingreso a la enseñanza de un dialecto desconocido a través de la interacción instructiva de los jóvenes chilenos, que se inicia en el segundo patrón de formación esencial (10-11 años) y prosigue hasta el cuarto año de secundaria (17). - 18 años de edad). La sustancia de autoridad pública anuncia que la motivación detrás del subsector de dialectos desconocidos en la Educación Secundaria es dotar a los estudiantes de un instrumento que les permita acceder a datos, información y avances, así como diferentes formas de vida, costumbres y perspectivas (Mineduc, 2020).

A esto se suma el desarrollo inventivo que está desarrollando el país. La organización de asociaciones de PC, por ejemplo, Internet es básica en varios deportes útiles y funciona. Además, ha surgido la necesidad de fusionar 
estos desarrollos en las prácticas informativas, como un segmento de nuevos métodos instructivos. Posteriormente, la utilización del desarrollo en la enseñanza del lenguaje es un factor de evidente aplicación en la realidad instructiva chilena. En consecuencia, es importante llegar a un arreglo que aborde otra reevaluación de las exhibiciones educativas que se entregan a partir de elementos genuinos reproducidos y que intentan sumergirse en mejores soportes especulativos y sensibles que directamente influyen en el aula. Con esta visión, el límite con respecto a la intervención ensayada por los sistemas de PC es enorme, ya que se trata de auténticos 'gadgets fonéticos' (López y Gallardo, 2005) aptos para mostrar una variedad de efectos sobre el aprendizaje (Mineduc, 2020).

En esta circunstancia concreta, surge la preocupación por conocer la perspectiva de dos animadores principales en la medida de educar y aprender: suplentes e instructores. Desde una perspectiva, se ha considerado aplicable para comprender las intuiciones de los educadores sobre el ciclo instructivo intervenido por los avances. Por otro, se propone comprender la impresión de estudiantes suplentes que buscan prepararse en la creación y comprensión del inglés, y que cuentan con métodos innovadores durante la interacción. Este artículo está dividido en cuatro partes fundamentales: comienza con el sistema hipotético y va seguido por el plan del examen, los resultados para incorporarlos en las últimas contemplaciones. El examen está incrustado en lo que respecta al proyecto FONDECYT (N 1120247) (Mineduc, 2020).

\section{¿Qué concebimos por e-learning?.}

La traducción literal al español nos llevaría a una concepción de “aprendizaje electrónico", no obstante Martín Hernández (2006) señala la concepción compleja del e-Learning que engloba aquellas aplicaciones y servicios que, tomando como base las TIC se orientan a facilitar el proceso de enseñanza-aprendizaje. Esta complejidad conceptual así como la diversidad de términos, conceptos y vocablos que se utilizan de forma indiscriminada para referirse a un sistema de formación singular, nos llevan a matizar lo que se entiende por e-Learning (Martín Hernández, 2006).

Una de las principales definiciones que se ha descubierto identificadas con e-learning es la creada por la Dirección General de Telecomunicaciones en 1996. Esta definición, a pesar de que no hace una referencia expresa al término e-adapting todavía utiliza la palabra teleeducación, mencionó cómo el avance de la medida de preparación a distancia (controlada o no dirigida) se puede caracterizar bajo esta marca, que, en vista de la utilización de innovaciones de datos y telemática, potencia la mejora de la educación, adaptable y abierto a descubrir cómo a cualquier beneficiario imaginable (Moptma, 1996 ). 
Azcorra (2001) comprende la teleformación o e-learning como una forma de aprendizaje a distancia de carácter abierto, inteligente y adaptable que se crea a través de los nuevos avances en datos y correspondencia, explotando lo más importante las ventajas que ofrece Internet. Por otra parte, Bartolomé (2004) llamó la atención sobre cómo el e-learning alude al avance de la preparación a distancia apoyado en los resultados potenciales instructivos que tienen las TIC, mientras que Area (2004) mostró que la preparación es un componente natural del e-learning, no una casualidad real entre instructor y suplente así como la utilización de las TIC para la mejora de la preparación.

Como se puede observar, el e-learning se ha relacionado de manera global con el avance de los encuentros intuitivos, abiertos y adaptables que no preparan ojo a ojo, que tienen en las TIC, y fundamentalmente en Internet, la ayuda crucial para el turno de trabajo. En este sentido, se coincide con Rosenberg (2001) al mostrar que el e-learning es un tipo de instrucción a distancia, pero que no toda la educación a distancia se compone de e-learning.

\section{E-learning y educación.}

La presentación del e-learning en la escuela va más allá de la presentación de las TIC en ciclos instructivos y tiene los aliados académicos fundamentales de la misma en los estándares de asociación y coherencia creados por Dewey. En esta línea, la acogida de las conjeturas e-learning supone una garantía para un modelo académico donde los estudiantes suplentes asumen una responsabilidad más destacada en la escolarización, sumando al avance de la competencia en la medida de aprendizaje instruccional y, por tanto, a la mejora subjetiva de lo instructivo (Dewey, 1938).

La regla de cooperación en la que se basa este modelo impulsa el avance de ciclos instructivos más individualizados y sinérgicos. En este sentido, se plantea la trascendencia de centrarse en los puntos de vista académicos sobre los mecánicos, a pesar de que se sabe que estos últimos condicionarán generalmente el cumplimiento de la proposición instructiva a crear. El ajuste del modelo instructivo que infiere el e-learning debe centrarse en los orígenes, objetivos y elecciones del instructor en un grado más prominente que en las TIC que se utilizarán; El cambio que implica el elearning no radica en trabajar con la medida del aprendizaje educar (García Aretio, 2002).

\section{E-learning y educación.}

La presentación del e-learning en la escuela va más allá de la presentación de las TIC en ciclos instructivos y tiene los principales aliados académicos de la misma en los estándares de colaboración y coherencia. En esta línea, la selección de conjeturas de e-learning da garantía a un modelo 
instructivo en el que los suplentes asuman una responsabilidad más destacada en la escolarización, sumando al avance de la eficacia en la medida de aprendizaje educativo y, por tanto, a la mejora subjetiva de la educación (Dewey, 1938).

El estándar de asociación en el que se basa este modelo impulsa el avance de ciclos instructivos más individualizados y sinérgicos. En este sentido, se llama la atención sobre la importancia de enfocar los ángulos instructivos sobre los mecánicos, a pesar de que se sabe que estos últimos generalmente condicionan el cumplimiento de la proposición instructiva a crear. El ajuste del modelo educativo que infiere el e-Learning debe centrarse en las organizaciones, objetivos y elecciones del instructor en un grado más notable que en las TIC que se utilizarán (García Aretio, 2002).

\section{Metodología}

Con el fin de determinar los aspectos relacionados con el análisis y evaluación del proceso enseñanza aprendizaje del idioma inglés en estudiantes de educación superior, se realizó encuestas dirigidas a estudiantes. Además, se consideraron dos puntos de vista interesantes: socios tecnológicos y educativos. Cabe señalar que estos métodos ayudan a establecer un marco de referencia para identificar y priorizar los factores clave en el sistema interactivo, comprender la dinámica y facilitar la toma de decisiones para resolver problemas que afectan a los participantes de la educación.

\section{Población}

En el presente trabajo de investigación la población de estudio son los 349 estudiantes del Instituto Superior Tecnológico Guayaquil (ISTG).

\section{Muestra}

Se realizó un muestreo no probabilístico por conveniencia a todos los estudiantes del Instituto Superior Tecnológico Guayaquil (ISTG).

\section{Resultados}

Ítem 1: El material educativo contribuye con mi aprendizaje en el idioma inglés

Tabla 1. Material Educativo

\begin{tabular}{lll}
\hline Respuestas & Frecuencia & Porcentaje \\
\hline Totalmente desacuerdo & 0 & $0,00 \%$ \\
En desacuerdo & 0 & $0,00 \%$ \\
Ni de acuerdo ni en desacuerdo & 4 & $1,15 \%$ \\
De acuerdo & 56 & $16,05 \%$ \\
Totalmente de acuerdo & 289 & $82,81 \%$ \\
Total & $\mathbf{3 4 9}$ & $\mathbf{1 0 0 , 0 0 \%}$ \\
\hline
\end{tabular}




\section{Interpretación:}

El material didáctico se suele emplear como nexo o elemento de unión entre el docente y/o discente y la realidad. Los resultados de la encuesta realizada a los estudiantes muestran que el $83 \%$ está totalmente de acuerdo con lo mencionado anteriormente, el $16 \%$ está de acuerdo y el $1 \%$ está ni de acuerdo ni en desacuerdo.

Ítem 2: Las sesiones virtuales me ayudan a comprender el curso de inglés

Tabla 2. Sesiones Virtuales

\begin{tabular}{lcc}
\hline Respuestas & Frecuencia & Porcentaje \\
\hline Totalmente desacuerdo & 0 & $0,00 \%$ \\
En desacuerdo & 0 & $0,00 \%$ \\
Ni de acuerdo ni en desacuerdo & 8 & $2,29 \%$ \\
De acuerdo & 40 & $11,46 \%$ \\
Totalmente de acuerdo & 301 & $86,25 \%$ \\
Total & $\mathbf{3 4 9}$ & $\mathbf{1 0 0 , 0 0 \%}$ \\
\hline
\end{tabular}

\section{Interpretación:}

Aprender inglés mediante cursos virtuales, da la oportunidad de estudiar en cualquier momento y en cualquier lugar, es por eso que permite a los estudiantes priorizar con mayor facilidad las vidas y se convierte en una excelente opción para aprender un nuevo idioma de manera eficaz. Así pues, se encuestó a los estudiantes del ISTG quienes el $86 \%$ mencionaron estar totalmente de acuerdo en que las sesiones virtuales les ayuda a comprender mejor el curso de inglés, el $12 \%$ está de acuerdo y el $2 \%$ está ni de acuerdo ni en desacuerdo.

Ítem 3: La comunicación con mi docente usando la plataforma es clara

Tabla 3. Comunicación con mi docente

\begin{tabular}{lcc}
\hline Respuestas & Frecuencia & Porcentaje \\
\hline Totalmente desacuerdo & 0 & $0,00 \%$ \\
En desacuerdo & 0 & $0,00 \%$ \\
Ni de acuerdo ni en desacuerdo & 8 & $2,29 \%$ \\
De acuerdo & 40 & $11,46 \%$ \\
Totalmente de acuerdo & 301 & $86,25 \%$ \\
Total & $\mathbf{3 4 9}$ & $\mathbf{1 0 0 , 0 0 \%}$ \\
\hline
\end{tabular}

\section{Interpretación:}

Las diferentes herramientas tecnológicas y plataformas facilitan la comunicación y el proceso de enseñanza-aprendizaje en los estudiantes, por esto se analizó a los estudiantes del ISTG a través de las encuestas en la cual el $86 \%$ mencionó estar totalmente de acuerdo con que la comunicación 
docente-estudiante es clara, el $12 \%$ está de acuerdo y el $2 \%$ está ni de acuerdo ni en desacuerdo.

Ítem 4: El trabajo en equipo se da forma organizada

Tabla 4. El trabajo en equipo se da forma organizada

\begin{tabular}{lcc}
\hline Respuestas & Frecuencia & Porcentaje \\
\hline Totalmente desacuerdo & 0 & $0,00 \%$ \\
En desacuerdo & 0 & $0,00 \%$ \\
Ni de acuerdo ni en desacuerdo & 24 & $6,88 \%$ \\
De acuerdo & 92 & $26,36 \%$ \\
Totalmente de acuerdo & 233 & $66,76 \%$ \\
Total & 349 & $100,00 \%$ \\
\hline
\end{tabular}

\section{Interpretación:}

En el trabajo en equipo las habilidades de los integrantes son complementarias, mantienen una responsabilidad individual y mutua. En consecuencia los resultados obtenidos de la encuesta muestran que el $67 \%$ de los estudiantes del ISTG está totalmente de acuerdo en que el trabajo efectuado en el mismo se da de forma organizada, el $26 \%$ está de acuerdo y el $7 \%$ está ni de acuerdo ni en desacuerdo.

Ítem 5: Los deberes se reparten de forma equitativa en el grupo de trabajo

Tabla 5. Los deberes se reparten de forma equitativa en el grupo de trabajo

\begin{tabular}{lcc}
\hline Respuestas & Frecuencia & Porcentaje \\
\hline Totalmente desacuerdo & 10 & $2,87 \%$ \\
En desacuerdo & 34 & $9,74 \%$ \\
Ni de acuerdo ni en desacuerdo & 50 & $14,33 \%$ \\
De acuerdo & 57 & $16,33 \%$ \\
Totalmente de acuerdo & 198 & $56,73 \%$ \\
Total & 349 & $100,00 \%$ \\
\hline
\end{tabular}

\section{Interpretación:}

En un salón de clases es estratégico distribuir a los alumnos en equipos de trabajo que incorporen diversas visiones, niveles y formas de trabajar. Mezclar alumnos capaces de ayudar al resto, estudiantes con dificultades y alumnos que se mantienen en la media del aula, de este modo unos aprenderán de otros y estarás aprovechando este potencial para que aprendan y contribuyan al resultado final con las fortalezas. Por consiguiente, los resultados demuestran que el $57 \%$ de los estudiantes consideran estar totalmente de acuerdo en que los docentes reparten tareas de una manera equitativa cuando forman grupos de trabajo, el 16\% está de acuerdo, el 14\% está ni de acuerdo ni en desacuerdo. No obstante el 10\% y 3\% está en desacuerdo y totalmente en desacuerdo respectivamente.

Ítem 6: Comparto mis ideas con los miembros de mi grupo de trabajo 
Tabla 6. Comparto mis ideas con los miembros de mi grupo de trabajo

\begin{tabular}{lcc}
\hline Respuestas & Frecuencia & Porcentaje \\
\hline Totalmente desacuerdo & 2 & $0,57 \%$ \\
En desacuerdo & 7 & $2,01 \%$ \\
Ni de acuerdo ni en desacuerdo & 12 & $3,44 \%$ \\
De acuerdo & 78 & $22,35 \%$ \\
Totalmente de acuerdo & 250 & $71,63 \%$ \\
Total & 349 & $100,00 \%$ \\
\hline
\end{tabular}

\section{Interpretación:}

En un grupo de trabajo en importante compartir y apreciar los diferentes puntos de vista de cada uno de los miembros, así pues lo afirman el $72 \%$ de los estudiantes que dicen estar totalmente de acuerdo con que esto si se lleva a cabo en la institución en estudio, el 22\% está de acuerdo y el $3 \%$ está ni de acuerdo ni en desacuerdo. No obstante, el $2 \%$ y $1 \%$ manifiestan estar en desacuerdo y totalmente en desacuerdo.

Ítem 7: Me siento motivado por mi docente durante las clases virtuales

Tabla 7. Motivación del Docente

\begin{tabular}{lcc}
\hline Respuestas & Frecuencia & Porcentaje \\
\hline Totalmente desacuerdo & 0 & $0,00 \%$ \\
En desacuerdo & 0 & $0,00 \%$ \\
Ni de acuerdo ni en desacuerdo & 13 & $3,72 \%$ \\
De acuerdo & 69 & $19,77 \%$ \\
Totalmente de acuerdo & 267 & $76,50 \%$ \\
Total & 349 & $100,00 \%$ \\
\hline
\end{tabular}

\section{Interpretación:}

La motivación posee una importancia fundamental para que el alumnado muestre interés en la educación y predisposición a aprender. Un alumno con motivación conseguirá buenos resultados y presentará un mayor interés por continuar con la etapa formativa y alcanzar metas cada vez de mayor complejidad. Los resultados de las encuestas muestran que el $77 \%$ está totalmente de acuerdo en que se sienten motivados por los docentes en las clases virtuales, el $20 \%$ está de acuerdo y el $4 \%$ está ni de acuerdo ni en desacuerdo.

Ítem 8: El docente resuelve mis dudas de manera oportuna 
Tabla 8. Dudas resueltas por los docentes

\begin{tabular}{lcc}
\hline Respuestas & Frecuencia & Porcentaje \\
\hline Totalmente desacuerdo & 0 & $0,00 \%$ \\
En desacuerdo & 0 & $0,00 \%$ \\
Ni de acuerdo ni en desacuerdo & 0 & $0,00 \%$ \\
De acuerdo & 47 & $13,47 \%$ \\
Totalmente de acuerdo & 302 & $86,53 \%$ \\
Total & 349 & $100,00 \%$ \\
\hline
\end{tabular}

\section{Interpretación:}

Los docentes con el uso adecuado de las herramientas apoyan de manera efectiva el aprendizaje de los estudiantes; sin embargo, también debe tener disponibilidad, atención e interés para resolver dudas académicas del alumnado, en la encuesta realizada a los estudiantes del ISTG se determinó que el $87 \%$ y $13 \%$ de los estudiantes están totalmente de acuerdo y de acuerdo respectivamente, en que en la institución los docentes están prestos a resolver cualquier duda que ellos tengan acerca de la clase que se imparte. Ítem 9: La retroalimentación del docente se da de forma efectiva

Tabla 9. Retroalimentación del Docente

\begin{tabular}{lcc}
\hline Respuestas & Frecuencia & Porcentaje \\
\hline Totalmente desacuerdo & 0 & $0,00 \%$ \\
En desacuerdo & 0 & $0,00 \%$ \\
Ni de acuerdo ni en desacuerdo & 3 & $0,86 \%$ \\
De acuerdo & 47 & $13,47 \%$ \\
Totalmente de acuerdo & 299 & $85,67 \%$ \\
Total & 349 & $100,00 \%$ \\
\hline
\end{tabular}

\section{Interpretación:}

El profesor debe permitir a los estudiantes experimentar y reflexionar sobre el objeto de aprendizaje, por esto se realizó una encuesta en la cual los estudiantes indicaron que el $86 \%$ está totalmente de acuerdo y el 14\% está de acuerdo en que los docentes realizan una adecuada retroalimentación de forma efectiva.

Ítem 10: La plataforma virtual es de un diseño amigable

Tabla 10. La plataforma virtual es de un diseño amigable

\begin{tabular}{lll}
\hline Respuestas & Frecuencia & Porcentaje \\
\hline Totalmente desacuerdo & 0 & $0,00 \%$ \\
En desacuerdo & 0 & $0,00 \%$ \\
Ni de acuerdo ni en desacuerdo & 7 & $2,01 \%$ \\
De acuerdo & 38 & $10,89 \%$ \\
Totalmente de acuerdo & 304 & $87,11 \%$ \\
Total & 349 & $100,00 \%$ \\
\hline
\end{tabular}




\section{Interpretación:}

Las plataformas virtuales son unos sistemas que nos permiten la ejecución de diversas aplicaciones en un mismo ambiente, permitiendo así que un usuario acceda a ella vía internet. En consecuencia, en la encuesta realizada se analizó que el $87 \%$ de los estudiantes están totalmente de acuerdo en que la plataforma virtual utilizada en la institución es de un diseño amigable, 11\% está de acuerdo y un $2 \%$ está ni de acuerdo ni en desacuerdo. Ítem 11: Hago uso de la plataforma educativa de manera constante

Tabla 11. Uso de la plataforma educativa

\begin{tabular}{lll}
\hline Respuestas & Frecuencia & Porcentaje \\
\hline Totalmente desacuerdo & 3 & $0,86 \%$ \\
En desacuerdo & 3 & $0,86 \%$ \\
Ni de acuerdo ni en desacuerdo & 12 & $3,44 \%$ \\
De acuerdo & 56 & $16,05 \%$ \\
Totalmente de acuerdo & 275 & $78,80 \%$ \\
Total & 349 & $100,00 \%$ \\
\hline
\end{tabular}

\section{Interpretación:}

El hecho de existir diversas plataformas educativas debe estar en constante movimiento o uso por parte de los estudiantes para que sean muy bien aprovechadas, en consecuencia, los estudiantes del ISTG mencionaron que el $79 \%$ está totalmente de acuerdo en que hacen uso constante de la plataforma educativa en la institución, el 16\% está de acuerdo, el $3 \%$ está ni de acuerdo ni en desacuerdo, el $1 \%$ y $1 \%$ está en desacuerdo y totalmente desacuerdo respectivamente recalcando no dar uso de la plataforma virtual. Ítem 12: Participo activamente durante las videoconferencias

Tabla 12. Participación activa en las videoconferencias

\begin{tabular}{lll}
\hline Respuestas & Frecuencia & Porcentaje \\
\hline Totalmente desacuerdo & 3 & $0,86 \%$ \\
En desacuerdo & 23 & $6,59 \%$ \\
Ni de acuerdo ni en desacuerdo & 39 & $11,17 \%$ \\
De acuerdo & 47 & $13,47 \%$ \\
Totalmente de acuerdo & 237 & $67,91 \%$ \\
Total & 349 & $100,00 \%$ \\
\hline
\end{tabular}

\section{Interpretación:}

La videoconferencia es una posibilidad técnica de los nuevos canales de comunicación, que permite la transmisión y recepción de información visual y auditiva. Por ello, los resultados muestran que el $68 \%$ está totalmente de acuerdo en que participan activamente durante las videoconferencias, el $13 \%$ está de acuerdo y el $11 \%$ ni de acuerdo ni en desacuerdo, el $7 \%$ está en desacuerdo y el $1 \%$ está totalmente en desacuerdo. 


\section{Conclusiones y Recomendaciones}

El desarrollo y evolución de las Tic y el E-Learning como una modalidad educativa en el proceso de enseñanza-aprendizaje del idioma inglés en estudiantes, si bien ha estado inspirada en la educación a distancia tradicional, hoy en día es la técnica mayormente utilizada y necesaria por los estudiantes en las diferentes instituciones educativas.

De acuerdo con lo analizado en la investigación, se trataría, por un lado, de una modalidad que en el transcurso del tiempo, el uso de las TIC facilitaría los procesos de enseñanza-aprendizaje en la universidad (nivel superior).

Por otro lado, se trata de una modalidad con amplias vías de fortalecimiento educativa, ante la necesidad de tener conocimientos permanentemente actualizados a lo largo de la vida, sin que necesariamente interfieran en nuestro diario vivir, ni requieran invertir una gran cantidad de tiempo.

En la actualidad el uso del E-Learning ha sido una herramienta fundamental para continuar con el proceso educativo a nivel universitario y con ello facilitar la formación de profesionales al servicio del desarrollo de los países.

Este tipo de enseñanza online permite la interacción del usuario con el material mediante la utilización de diversas herramientas informáticas. En este sentido, será muy interesante incentivar el uso de las TIC y el E-Learning, a través de las cuales se puede facilitar de forma rápida y sencilla la comunicación y el acceso a diferentes fuentes de información.

\section{References:}

1. Albion. (2015). Teachers' professional development for ICT integration: Towards a reciprocal relationship between research and practice. RIDE Revista Iberoamericana para la Investigación y el Desarrollo Educativo.

2. Alsabawy, \& Cater-Steel. (2013). IT infrastructure services as a requirement for E-Learning system sucess.Computers \& Education. Scielo.

3. Álvarez. (2012). Sistema de Gestión de Aprendizaje. Obtenido de http://es.scribd.com/doc/100356593/3-Sistemas-de-Gestion-deAprendizaje-v21.

4. Arancibia, Contreras, \& Soto. (2010). Concepciones del profesor sobre el uso educativo de las tecnologías de la información y la comunicación (TIC) asociadas a procesos de enseñanza-aprendizaje en el aula escolar. Scielo.

5. Area. (2004). Los medios y las tecnologías en la educación. Madrid: Ediciones Pirámide. 
6. Asongu. (2017). Enhancing ICT for inclusive human development in Sub-Saharan Africa, Technological Forecasting and Social Change.

7. Ausín, V., Abella, V., Delgado, \& Hortigüela. (2016). Aprendizaje Basado en Proyectos a través de las TIC. Una Experiencia de Innovación Docente desde las Aulas Universitarias. Scielo.

8. Azcorra. (2001). Informe sobre el estado de a teleeducación en España. Madrid: Asociación de Usuarios de Internet.

9. Baldassar. (2016). Wilding, ICT-based co-presence in transnational families and communities: challenging the premise of face-to-face proximity in sustaining relationships. Global Networks.

10. Baller, S., Dutta, S., \& Lanvín , B. (2016). Global information technology report 2016. Geneva, Ouranos.

11. Bartolomé. (2004). Conceptos Básicos. Conceptos Básicos. Revista de medios y educación, 7-20.

12. Bello . (2018). Digital skills in young people entering the university: realities to innovate in university education . RIDE Revista Iberoamericana para la Investigación y el Desarrollo Educativo.

13. Cabero, J. (2010). Los retos de la integración de las TIC's en los procesos educativos, Límites y posibilidades, Perspectiva Educacional, Formación de Profesores. Scielo.

14. Cabero, Llorente, \& Román. (2015). Las Herramientas de Comunicación en el Aprendizaje Mezclado. Universidad de Sevilla. Obtenido de http://www.lmi.ub.es/te/any2004/documentacion/3_cabero.pdf.

15. CEPAL-UNESCO. (2020). ¿Cómo estás aprendiendo durante la pandemia de COVID-19? Obtenido de https://es.unesco.educationresponse.

16. Deveci. (2016). Examination of University Students' Level of Satisfaction and Readiness for E-Courses and the Relationship between Them. European Journal of Contemporary Education.

17. Dewey. (1938). Democracy and education: an introduction to the philosophy of education. New York: Macmillan.

18. García Aretio. (2002). Aprendizaje y Tecnologías Digitales ¿Novedad o Innovación? Obtenido de http://ipes.anep.edu.uy/.

19. Herrera, A. (2015). Una mirada reflexiva sobre las TIC en Educación Superior. Revista electrónica de investigación educativa.

20. Hodges. (2020). The Difference Between Emergency Remote Teaching and Online Learning. EduCause Review.

21. Horacio, Ortiz, \& Bergamaschi. (2020). La educación en tiempos de coronavirus. Banco Interamericano de Desarrollo.

22. Koh. (2015). Technological Pedagogical Content Knowledge (TPACK) and design thinking: A framework to support ICT lesson 
design for 21 st century learning. The Asia-Pacific Education Researcher.

23. López , S., \& Hernández. (2016). Principios para elaborar un modelo pedagógico universitario basado en las TIC, Estado del arte, EPISTEME. Revista digital de ciencia, Tecnología e Innovación.

24. Marín, Inciarte, \& Hernández. (2017). Estrategias de las Instituciones de Educación Superior para la Integración de las Tecnología de la Información y la Comunicación y de la Innovación en los Procesos de Enseñanza, Un Estudio en el Distrito de Barranquilla, Colombia. Formación Universitaria.

25. Marshall. (2012). Improving the quality of E-Learning: lessons from the eMM. Journal of Computer Assisted Learning.

26. Martín Hernández. (2006). Conceptos en La formación sin distancia. Madrid: Editorial De Alvarado y Rodríguez.

27. Mineduc. (2020). Objetivos Fundamentales y Contenidos Mínimos Obligatorios de la Educación Media. Obtenido de https://www.mineduc.cl/.

28. Moptma. (1996 ). Estudio técnico para la elaboración de un plan de acción para la Administración destinado a impulsar la tele-educación. Ministerio de Obras Públicas.

29. Pérez, \& Olguín. (2018). The Role of Planning and Implementation of ICT in Operational Benefits, Sustainability. Scielo.

30. Prensky. (2001). Digital Natives, Digital Immigrants, On the Horizon. University Press.

31. Rosenberg. (2001). E-learning: strategies for delivering knowledge in the digital age. . New York: McGraw-Hill.

32. Sanz, D., \& Crissien-Borrero, T. (2018). Responsabilidad en las instituciones de educación superior, Cultura, Educación y Sociedad. UT Riviera Maya.

33. UNESCO. (2013). Enfoques estratégicos sobre las TICs en educación en America Latina y el Caribe. Organización de las Naciones Unidas para la Educación, la Ciencia y la Cultura.

34. Zempoalteca, Barragán, \& González. (2017). Formación en TIC y competencia digital en la docencia en instituciones públicas de educación superior. Scielo. 